\title{
the relativity of hyperbolic space
}

\author{
B. H. Lavenda* \\ Università degli Studi, Camerino 62032 (MC) Italy
}

(Dated: January 29, 2009)

\begin{abstract}
Paths of shortest length, or geodesics, may not appear as straight lines because acceleration creates distortion. For spaces of constant curvature there are only two possibilities: Either rulers get longer as they move away from the origin or they shrink. Because the longitudinal Doppler shift corresponds to the measure of distance, in velocity space, this space is hyperbolic, corresponding to the second of the two possibilities. Transformations from one inertial frame to another are related to geometrical rigid motions. The square root of the Doppler shift is related to transformations of uniformly accelerated frames. Applications to general relativity and cosmology are given including the time-velocity metric of the Friedmann universe of dust at zero pressure, Hubble's law and the exponential red-shift.
\end{abstract}

"Of course, since Einstein, we do not use hyperbolic geometry to model the geometry of the universe" [8].

\section{INTRODUCTION}

Almost immediately after the birth of special relativity Sommerfeld [23] made the interesting observation that the relativistic composition laws of velocities are "no longer the formulas of the plane but those of spherical trigonometry (with imaginary sides)," trigonometrical formulas obtained by replacing the real argument by an imaginary one. Spheres of imaginary radius had been known for a long time, as this identity was pointed out by Lobachevsky himself [12]. The first explicit connection of Lobachevsky geometry to relativity was made by Varićak [27]. The hyperbolic $(h)$ geometry of relativity represents the velocity addition law as a triangle on the surface of a pseudosphere, a surface of revolution looking like a bugle, and the angle of parallelism which measures the deviation from Euclidean (e) space. As the relative velocity approaches unity, the angle of parallelism approaches zero. The fact that the angle of parallelism provides a unique relation between circular and $h$-functions can be found in the early textbook on relativity by Silberstein [22]. These developments did not have a follow up, and no place for $h$-geometry could be found in the relativity textbooks that followed, with a single exception [26]. Undoubtedly, this was due to the influence of Einstein's general theory which is based upon Riemann geometry, where the presence of matter creates a local positive curvature.

Yet, even more astonishing is the part that Poincaré missed in all this. Over fifty years after the discovery of the hyperbolic geometry, Poincaré developed two models of $h$-geometry: The upper half-plane and disc models. The inhabitants of $h$-plane, known as Poincarites [16], consider geodesics as straight lines while to us Euclidean they would appear as circular arcs meeting the boundary orthogonally in the disc model, or see Poincarites shrink as they approach the real axis from the upper half-plane. Poincarites would not be able to measure their shrinkage because the ruler they use shrink along with them.

These distortions are created by motion, and Poincaré was well aware of the contraction that bodies undergo in the direction of the Earth's motion, of an amount proportional to the square of the aberration. This is the famous FitzGerald-Lorentz (FL) contraction that was first postulated independently by FitzGerald and Lorentz, as an explanation of the Michelson-Morley null result. Poincaré was also aware of the relativistic velocity composition law, since it was he who discovered it [18]. Yet, he did not recognize the relation between this composition law and the way $h$-distance is defined as the logarithm of the cross-ratio.

The question arose in projective geometry as to what remains invariant in a projection since lengths and angles do not. Since three points on a line are not invariant, because it is possible to project them on to another line, the minimum number points needed is four. But then, any other four points projectively related to the original ones will have the same cross-ratio since a projectivity is the product of a sequence of perspectivities. The invariant cross-ratio is related to the $h$-length through its logarithm. We will evaluate the $h$-measure of length in terms of its $e$-measure through the definition of the cross-ratio.

Measurements in relativity consist in sending and receiving light signals. Distances are measured in terms of time 
differences. At each step, the time it takes to receive a signal sent out at a previous time is that time times a factor which turns out to be the longitudinal Doppler shift [28]. The space-time transformations from one inertial frame to another, involving Doppler shifts, combine to give the Lorentz transformation [14]. Had Poincaré realized that his definition of $h$-distance in terms of the logarithm of the cross-ratio, which for the distance between any two velocity points on a vertical half-line with an endpoint at infinity, is proportional to the logarithm of the longitudinal Doppler shift, he could have carried over the battery of concepts and tools he developed some twenty years earlier to relativity-without distinguishing between the 'special' and 'general' theories of Einstein. This we plan to do in this paper.

The paper is organized as follows. We first discuss the connection between geometrical rigid motions and their relations to particular inertial frames of reference. Compounding Doppler shifts at different velocities yields the Poincaré composition law, which, in terms of homogeneous coordinates shows that the Lorentz transform is a unique Möbius automorphism which exchanges an inertial frame of equal and opposite velocities with the state at rest. We will also be appreciate it as the isomorphism the converts the Poincaré to the Klein models of the $h$-plane, as well as establishing the limit for $h$-rotations in terms of the angle of parallelism.

We then go on to discuss the relativistic phenomenon of aberration and show that it conforms to the $h$-law of sines. In terms of a right-triangle inscribed in a unit disc, angular deformations of the non-central angle and contractions of the side of the triangle perpendicular to the motion will be related to the fact that the sum of the angles of a $h$-triangle is less than $\pi$, and a FL contraction in the direction normal to the motion making it look like more of a rotation than a contraction, respectively.

The radar method of sending and receiving light signals to measure elapses in time and distance will then be used to contrast states of uniform motion and uniform acceleration. We confirm Whitrow's [29] conclusion that acceleration does, indeed, affect the rate of a clock, and convert his inequality for time dilatation into an equality for systems in uniform acceleration. The characteristic means that we find for times of reflection for systems in uniform motion and uniform acceleration imply different temporal scales.

Applications to general relativity and cosmology follow. Beltrami coordinates and logarithmic time are used to derive a metric first investigated by Friedmann which corresponds to 'dust-like' matter at zero pressure in terms of Einstein's mass tensor. The Friedmann-Lobachevsky space found confirmation in Hubble's discovery of the red-shift in the spectra of galaxies: The greater the shift the more distant the galaxy. This we show is a consequence of the $h$-measure of the velocity and its relation to the logarithmic scale of time through Hubble's law. A comparison with the 'general' relativity then follows, followed by a presentation of our major results.

\section{HYPERBOLIC RIGID MOTIONS AND INERTIAL REFERENCE FRAMES}

\section{Doppler generation of Möbius transformations}

It has long been known [22] that the relativistic composition of velocities obeys $h$-geometry. Robb [21] proposed to call the $e$-measure of the velocity

$$
\bar{u}=\tanh u
$$

the 'rapidity,' where $\bar{u}$ is the relative velocity, having set the absolute constant $c=1$. We can invert (1) to find the expression

$$
u=\frac{1}{2} \ln \left(\frac{1+\bar{u}}{1-\bar{u}}\right)=\frac{1}{2} \ln \{-1,1 \mid u, 0\},
$$

for the $h$-measure of the relative velocity $u$, which unlike its $e$-counterpart is not confined to the closed interval $[-1,1]$. The bracket represents the cross-ratio of the distance between $x$ and $y$ in the closed interval $[a, b]$

$$
\{a, b \mid x, y\}=\frac{(a-x)(b-y)}{(a-y)(b-x)} .
$$

Exponentiating both sides of (2) shows that the exponential of the $h$-length is given by the longitudinal Doppler shift

$$
e^{u}=\left(\frac{1+\bar{u}}{1-\bar{u}}\right)^{1 / 2}=: K
$$


We will now show that compounding Doppler shifts generates Möbius transforms thereby relating geometric rigid motions with specific inertial frames.

As a preliminary, compounding the Doppler shift at velocity $\bar{u}$ with itself gives

$$
\left(\frac{1+\bar{u}}{1-\bar{u}}\right)=\left(\frac{1+\lambda}{1-\lambda}\right)^{1 / 2}
$$

where $\lambda$ is the relative velocity in an inertial frame comprised of equal and opposite velocities

$$
\lambda=\frac{2 \bar{u}}{1+\bar{u}^{2}}=\tanh (2 u)
$$

Next, consider the cross-ratio

$$
\{-1,1 \mid \bar{v}, \lambda\}=\frac{1+\bar{v}}{1-\bar{v}} \cdot \frac{1-\lambda}{1+\lambda}=\frac{1+\bar{v}^{\prime}}{1-\bar{v}^{\prime}} .
$$

The 'new' relativity velocity $\bar{v}^{\prime}$ is given in terms of the 'old' by

$$
\bar{v}^{\prime}=\frac{\bar{v}-\lambda}{1-\lambda \bar{v}}
$$

which will be easily recognized as the Poincaré composition law of the velocities. It is quite surprising that he did not make the connection with the cross-ratio and his models of the $h$-plane. Introducing the second equality in (5) in (6) gives the familiar Lorentz 'rotation'

$$
\bar{v}=\frac{\bar{v}^{\prime} \cosh (2 u)+\sinh (2 u)}{\bar{v}^{\prime} \sinh (2 u)+\cosh (2 u)},
$$

in terms of the homogeneous coordinates $\bar{v}$ and $\bar{v}^{\prime}$.

Multiplying (6) out gives

$$
\bar{v}^{\prime} \bar{v}+\lambda^{-1}\left(\bar{v}-\bar{v}^{\prime}\right)-1=0
$$

Two cases are of interest: If the relative velocities are equal, $\bar{v}=\bar{v}^{\prime},(7)$ becomes the simplest $h$-involution with conjugate points at \pm 1 . This is not of physical interest; rather, what is of physical interest is when the velocities are equal and opposite, $\bar{v}=-\bar{v}^{\prime}$. For then (7) reduces to the quadratic form

$$
\bar{v}^{2}-2 \lambda^{-1} \bar{v}+1=0
$$

which has two real roots

$$
\bar{v}_{ \pm}=\frac{1 \pm \sqrt{ }\left(1-\lambda^{2}\right)}{\lambda}
$$

Since the relative velocity $\lambda$ is given by (5) the two roots are $\bar{v}_{+}=1 / \bar{u}$ and $\bar{v}_{-}=\bar{u}$.

The negative of $(6)$

$$
\mathcal{M}_{\lambda}(\bar{v})=\frac{\bar{v}-\lambda}{\lambda \bar{v}-1}=\{-1,1 \mid \bar{v}, \lambda\}
$$

is the unique Möbius automorphism which exchanges $\lambda$ and 0 , viz., $\mathcal{M}_{\lambda}(\lambda)=0$ and $\mathcal{M}_{\lambda}(0)=\lambda$. This can be recognized as a special case of the property that $\mathcal{M}_{\lambda}$ is involutory: $\mathcal{M}_{\lambda} \circ \mathcal{M}_{\lambda}=\mathcal{I}$, the identity.

A Möbius transform takes any triplet $\left(\bar{v}_{1}, \bar{v}_{2}, \bar{v}_{3}\right)$ into any other triplet. The demonstration of the existence of such a transform rests on showing that there is a Möbius transform for which $\mathcal{M}\left(\bar{v}_{1}\right)=0, \mathcal{M}\left(\bar{v}_{2}\right)=1$ and $\mathcal{M}\left(\bar{v}_{3}\right)=\infty$. The values are not only the range of the conjugate points, $\bar{u}$ and $1 / \bar{u}$, but, moreover, allow the Möbius transform to be written as the invariant cross-ratio

$$
\mathcal{M}(\bar{v})=\left\{\bar{v}, \bar{v}_{2} \mid \bar{v}_{1}, \bar{v}_{3}\right\}=\frac{\left(\bar{v}-\bar{v}_{1}\right)}{\left(\bar{v}-\bar{v}_{3}\right)} \cdot \frac{\left(\bar{v}_{2}-\bar{v}_{3}\right)}{\left(\bar{v}_{2}-\bar{v}_{1}\right)} .
$$

Thus, by the construction of the cross-ratio, we have $\mathcal{M}\left(\bar{v}_{1}\right)=0, \mathcal{M}\left(\bar{v}_{2}\right)=1$, and $\mathcal{M}\left(\bar{v}_{3}\right)=\infty$. 
If we identify $\mathcal{M}$ with (8) then the triplet $(0,1, \infty)$ occurs when $\bar{v}_{1}=\lambda, \bar{v}_{2}=1$, and $\bar{v}_{3}=1 / \lambda$. The latter would imply that $\bar{v}$ is unbounded which is contradictory to special relativity. It is the symmetry of the $h$-involution which implies that if $\bar{u}$ is a solution, then so is $1 / \bar{u}$. We must therefore now show that the conjugate point $\bar{v}_{+}$is a repulsive fixed point, implying that repeated mappings of $\mathcal{M}_{\lambda}$ repel it away from $\bar{v}_{+}$. The Möbius transform $F$ conjugating the normalized $\mathcal{M}_{\lambda}$ to its standard form sends $\bar{v}_{+}$to zero and $\bar{v}_{-}$to infinity

$$
F(\bar{v})=\frac{\bar{v}-\bar{v}_{+}}{\bar{v}-\bar{v}_{-}}
$$

with

$$
F^{-1}(\bar{v})=\frac{-\bar{v}_{-} \bar{v}+\bar{v}_{+}}{-\bar{v}+1}
$$

Rather than calculate the composition $F \circ \mathcal{M}_{\lambda} \circ F^{-1}(z)$ to find the standard form of $\mathcal{M}_{\lambda}$, it suffices to calculate it at a point $F^{-1}(1)=\infty$ so that $\mathcal{M}_{\lambda}(\infty)=1 / \lambda$, and $F(1 / \lambda)=-1$. Thus,

$$
F \circ \mathcal{M}_{\lambda} \circ F^{-1}(\bar{v})=e^{i \pi} \bar{v}
$$

is the standard form of $\mathcal{M}_{\lambda}$, and represents a rotation about the fixed point $\bar{v}_{-}$which interchanges $\lambda$ with $O$.

The Möbius transformation (8) is therefore elliptic. Several properties are immediate: $\bar{v}_{+}$is the inverse of $\bar{v}_{-}$with respect to the circle of inversion $C_{1}$ in Fig. 1.

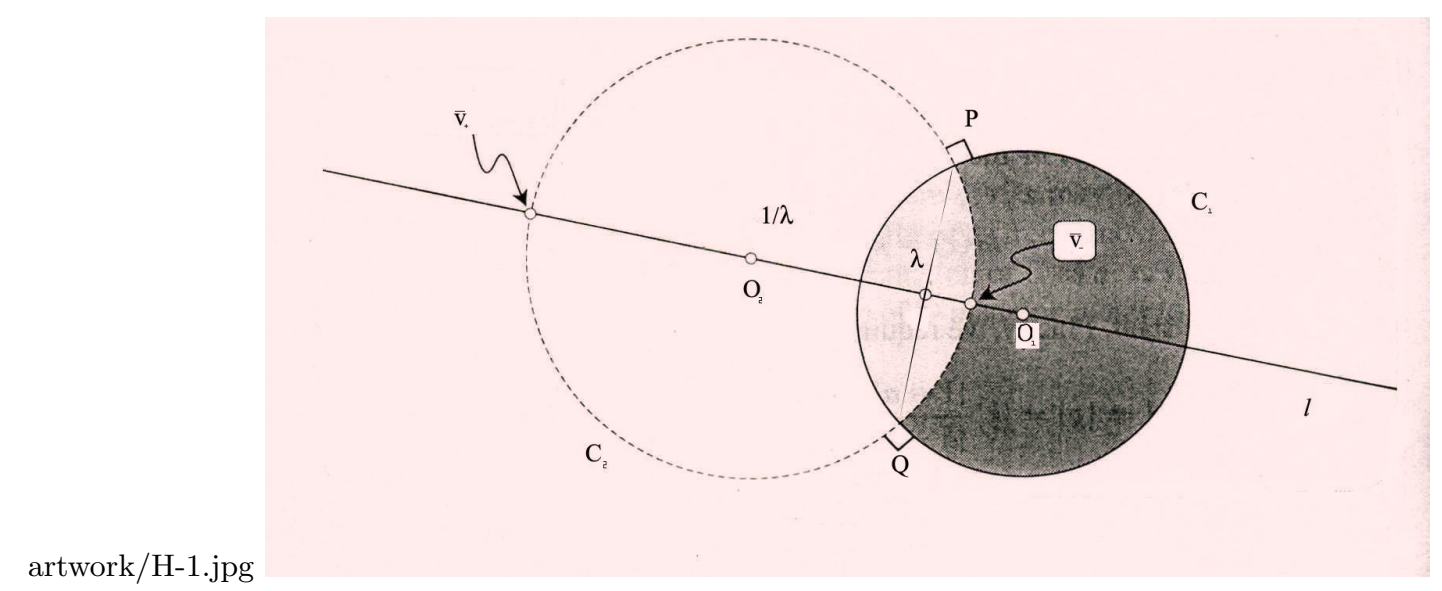

FIG. 1: Circles of inversion. The circle $C_{1}$ cuts the circle of inversion $C_{2}$ at right angles at $P$ and $Q$. A line from the origin of $C_{1}$ intersects $C_{2}$ at two points: $\bar{v}_{-}$and its inverse $\bar{v}_{+}$, which are fixed of the Lorentz transform. The relative velocity $\lambda$ lies at an equal $h$-distance from $\bar{v}_{-}$that $\bar{v}_{-}$lies from the origin. An $h$-rotation of $\pi$ occurs about $\bar{v}_{-}$which exchanges the state of uniform velocity $\lambda$ and the state of rest at $O_{1}$.

The fixed point $\bar{v}_{-}$lies inside the unit disc and the other fixed point $\bar{v}_{+}$lies outside except when $\bar{v}_{-}=\bar{v}_{+}$, and then they both lie on the circle of inversion. Fixed points closer and closer to the center send their conjugates points further and further away. Inversion takes circles orthogonal to the original one into themselves. Two such orthogonal circles, $C_{1}$ and $C_{2}$, are shown in Fig. 1 . The line $\ell$ joining their centers has the fixed points diametrically opposite lying on the circumference of the circle $C_{2}$ whose center is $O_{2}=\lambda^{-1}$. The arcs of $C_{2}$ lying in $C_{1}$, and orthogonal to it at the points of contact correspond to geodesics in the Poincaré model.

The intersection of the arc $P Q$ with the line $\ell$, shown in Fig. 2, occurs at the $h$-midpoint,

$\bar{v}_{-}=\bar{u}$, between 0 and $\lambda$. This is a direct consequence of the definition of $h$-distances: Whereas the $h$-distance from 0 to $\lambda$ is

$$
h(0, \lambda):=\{-1,1 \mid \lambda, 0\}=\ln \left(\frac{1+\lambda}{1-\lambda}\right)=2 \ln \left(\frac{1+\bar{u}}{1-\bar{u}}\right)
$$

the distance from 0 to $\bar{v}_{-}$is half as great

$$
h(0, \bar{u})=\{-1,1 \mid \bar{u}, 0\}=\ln \left(\frac{1+\bar{u}}{1-\bar{u}}\right) .
$$




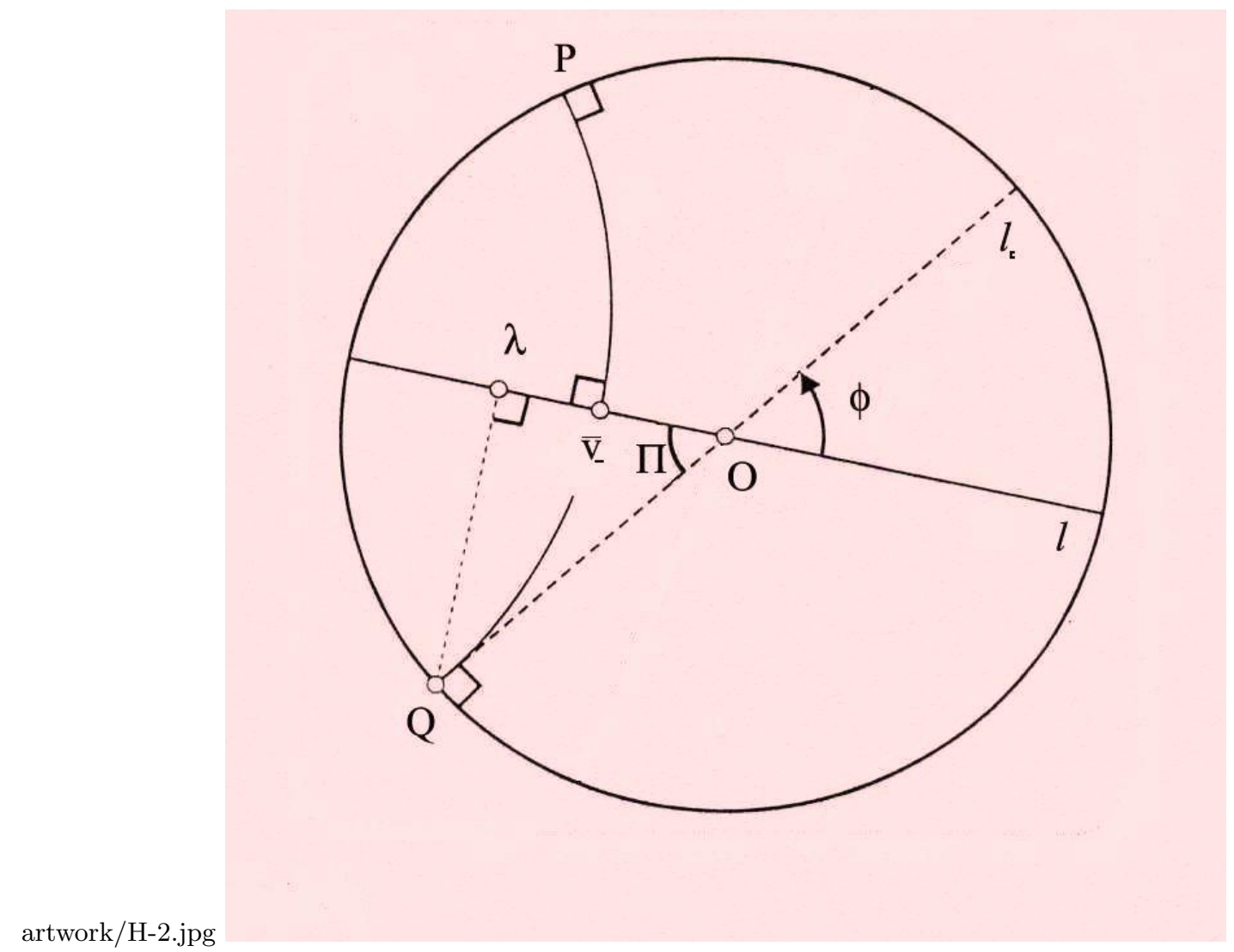

FIG. 2: A more detailed description of the circle containing the fixed point $\bar{v}_{1}$ and $\lambda$ which are uniform states of motion at relative velocities $\bar{u}$ and $2 \bar{u} /\left(1+\bar{u}^{2}\right)$. The Möbius automorphism of the disc may be considered as a composition of two $h$-rotations: A rotation of $\pi$ about the $h$-midpoint between the origin and $\lambda$, and a rotation about the origin. The maximum angle $\phi$ is determined by the angle of parallelism, $\Pi$, beyond which no motion can occur.

The $\operatorname{arc} P Q$ is itself the perpendicular bisector of $O \lambda$. The Möbius transformation (8) is the composition of two $h$-reflections in perpendicular lines through $\bar{v}_{-}$. Moreover, it is the unique Möbius automorphism that exchanges $O$ and $\lambda$ by a rotation through $\pi$ about the $h$-midpoint $\bar{v}_{-}$of the $h$-line segment.

Expression (5) is the isomorphism that takes the Poincaré model to the Klein model. Both are $h$-models of the unit disc, but whereas the Poincaré model is conformal the Klein model is not. The price to be paid is that geodesics in the Poincaré model are arcs of circles that cut the unit disc orthogonally, while the geodesics in the Klein model are straight lines. The isomorphism $\lambda$ maps the arc with ends $P$ and $Q$ onto the open chord with the same endpoints. Since $\bar{v}_{-}$is the point where line $\ell$ cuts the circumference of the orthogonal circle $C_{2}$, then $\lambda\left(\bar{v}_{2}\right)=\lambda(\bar{u})$ is the point at which the line $\ell$ hits the chord $Q$. But this is precisely the definition (5) of $\lambda$.

When the orthogonal arcs intersect, there occurs a $h$-rotation. At the limit when they are asymptotic, there is a limit rotation while when they become ultra-parallel there is an $h$-translation. However, $h$-translations in $h$-velocity space would contradict the fact that the limiting velocity is that of light. Consider the right-triangle $O \lambda Q$ formed by the intersection of lines $\ell$ and $\ell_{c}$. The angle at the origin $\Pi$ is the limiting angle, or the angle of 'parallelism.' For angles less than $\varphi$, the angle of parallelism will not be reached. Consequently, $\ell_{c}$ is the limiting line for $h$-rotations. According to the right-triangle, $\cos \Pi=\lambda$, while according to the Bolyai-Lobachevsky formula for the radian measure of the angle of parallelism

$$
\Pi(\lambda)=2 \tan ^{-1} e^{-\lambda} .
$$

The angle $\Pi(\lambda)$ is a function of the distance $\lambda$. The closer it is to $\pi / 2$, the less pronounced become the $h$-distortions. Thus,

$$
\cos \Pi(\lambda)=\tanh \lambda=\frac{2 \bar{v}_{-}}{1+\bar{v}_{-}^{2}}
$$


is just the isomorphism of the Poincaré model onto the Klein model. Since $\Pi$ must necessarily be acute, $h$-translations are ruled out, and the limiting rotation occurs for asymptotics.

\section{GEOMETRY OF DOPPLER AND ABERRATION PHENOMENA}

Consider the triangle formed by rotating $\bar{u}$ through an angle $\vartheta$, as shown in Fig. 3 .

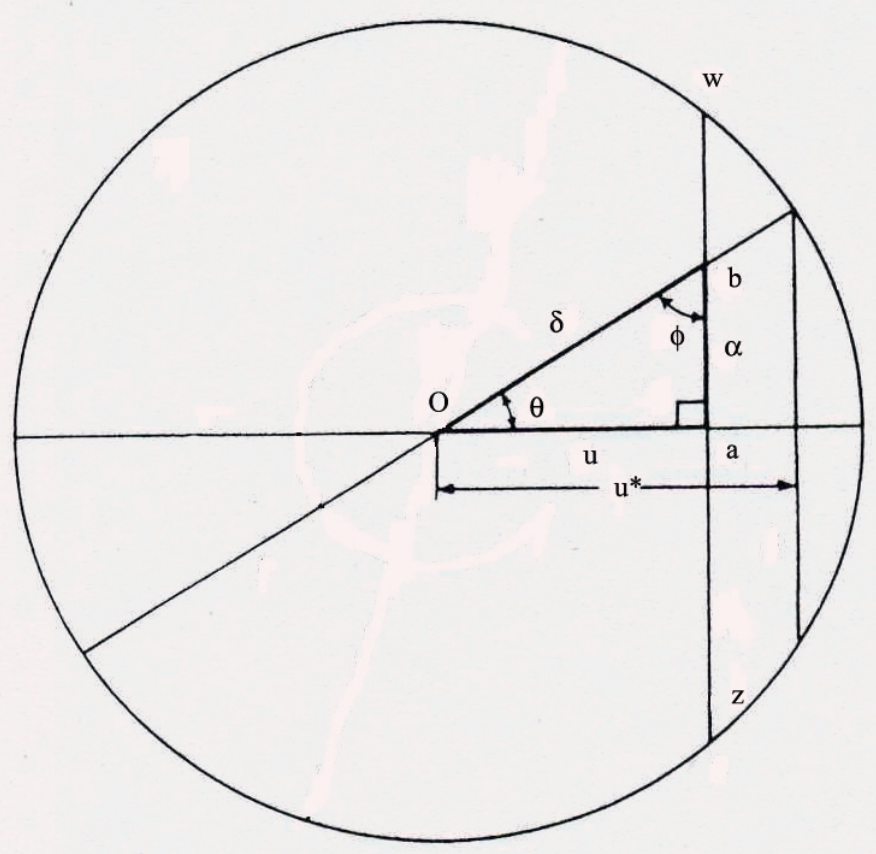

artwork/H-3.jpg

FIG. 3: A right triangle in a unit disc. Since angle $\vartheta$ is at the origin, there will be no difference between its $e$ - and $h$-measures. Due to distortion by the motion, the two measures of the angle $\varphi$ will no coincide. The critical velocity $\bar{u}$ will be reached when the vertical $w z$ is displaced to the right making the hypoteneuse $\delta=1$. The angle $\vartheta$ then becomes the angle of parallelism.

Rotations about the origin do not cause deformations, and there is no difference between $e$ - and $h$-measures of the angle. The right triangle has a hypotenuse $\delta$ and height $\alpha$. The cosine of the angle is the same in both measures

$$
\cos \vartheta=\cos \bar{\vartheta}=\bar{u} / \bar{\delta}=\tanh u / \tanh \delta
$$

However, the opposite angle $\varphi$ will undergo a contraction so that it will only be true that

$$
\cos \varphi=\bar{\alpha} / \bar{\delta}=\tanh \alpha / \tanh \delta .
$$

In order to determine the relation between $\varphi$ and $\bar{\varphi}$, it is necessary to calculate the height $\alpha$. If $w$ and $z$ are the corresponding ideal points by extending the $e$-length of height of the triangle, $e(a, b)$, so that it cuts the circle then the cross-ratio is

$$
\{a, b \mid w, z\}=\frac{e(a, w) e(b, z)}{e(a, z) e(b, w)}=\frac{\sqrt{ }\left(1-\bar{u}^{2}\right) \cdot\left(\sqrt{ }\left(1-\bar{u}^{2}\right)+\bar{\alpha}\right)}{\sqrt{ }\left(1-\bar{u}^{2}\right) \cdot\left(\sqrt{ }\left(1-\bar{u}^{2}\right)-\bar{\alpha}\right)} .
$$

We thus find the $e$-height in terms of the $h$-measure of height as

$$
\bar{\alpha}=\gamma^{-1} \tanh \alpha
$$

If the rotation occurred about the origin then $\bar{\alpha}$ would have been $\tanh \alpha$. But, because the motion is not at the origin, the $e$-length will appear contracted by a factor $\gamma^{-1}$. It is precisely this contraction which is responsible for the 
triangle defect in $h$-geometry

$$
\cos \bar{\varphi}=\bar{\alpha} / \bar{\delta}=\gamma^{-1} \frac{\tanh \alpha}{\tanh \delta}=\gamma^{-1} \cos \varphi
$$

Since the cosine is a decreasing function on the open interval $(0, \pi)$, it follows that $\varphi<\bar{\varphi}$, and this is the origin of the triangle defect in $h$-triangles. It is caused by the motion perpendicular to the direction of motion. This is the origin of the FL contraction in the direction perpendicular to the motion [10].

In $h$-geometry, the Pythagorean theorem, $\bar{\delta}^{2}=\bar{\alpha}^{2}+\bar{u}^{2}$, is converted into

$$
\cosh \delta=\cosh \alpha \cosh u
$$

because

$$
\begin{gathered}
\tanh ^{2} \delta=\tanh ^{2} \alpha+\tanh ^{2} u \\
\operatorname{sech}^{2} \delta=\operatorname{sech}^{2} \alpha+\operatorname{sech}^{2} u,
\end{gathered}
$$

and both sech and cosh are both positive functions. The $h$-Pythagorean theorem can be used in

$$
\sin \vartheta=\bar{\alpha} / \bar{\delta}=\gamma^{-1} \tanh \alpha / \tanh \delta,
$$

to get

$$
\sin \vartheta=\frac{\sinh \alpha}{\sinh \delta}
$$

In the limits $\alpha, \delta \rightarrow \infty$, such that their difference $\delta-\alpha$ is a positive constant,

$$
\bar{\alpha}=\gamma^{-1}=1 / \cosh \beta=\sin \vartheta=e^{\alpha-\delta} .
$$

We can consider a more general triangle in Fig. 4 with sides $\bar{\alpha}$ and $\bar{\delta}$, and base $\bar{u}$. The altitude $\bar{h}$ cuts the base

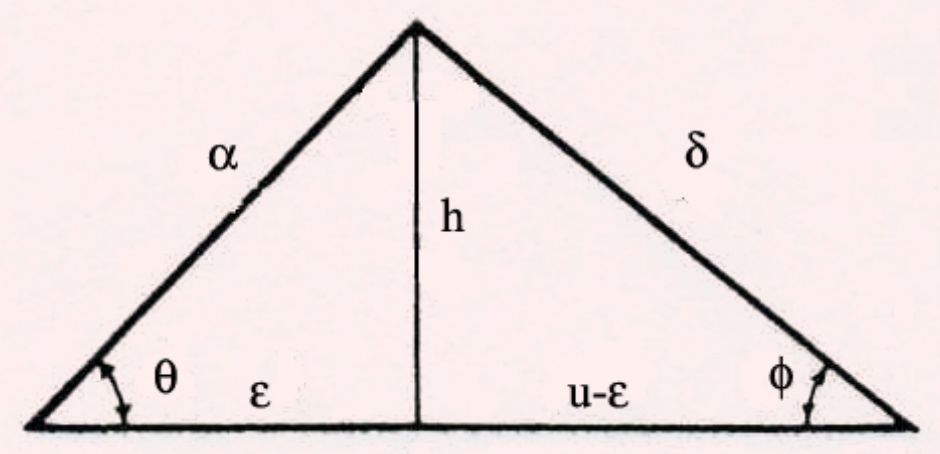

u

artwork/H-4.jpg

FIG. 4: Extension of hyperbolic trigonometry to general triangles.

into two parts $\bar{\varepsilon}$ and $\bar{u}-\bar{\varepsilon}$. The angles formed from the sides and the base are $\vartheta$ and $\varphi$. The sines of these angles $\operatorname{are} \sin \vartheta=\bar{h} / \bar{\alpha}=\tanh h \operatorname{sech} \varepsilon / \tanh \alpha=\sinh h / \sinh \alpha$, and $\sin \varphi=\bar{h} / \bar{\delta}=\tanh h \operatorname{sech}(u-\varepsilon) / \tanh \delta=\sinh h / \sinh \delta$, since deformation only occurs normal to the direction of motion, i.e., $\bar{u}$. Introducing the $h$-Pythagorean theorem of the first triangle

$\cosh \alpha=\cosh \varepsilon \cosh h$ 
into the $h$-Pythagorean theorem for the second triangle

$$
\cosh \delta=\cosh h \cosh (u-\varepsilon)=\cosh h(\cosh u / \cosh \varepsilon-\sinh \varepsilon \sinh u),
$$

results in

$$
\cosh \delta=\cosh \alpha / \cosh u-\tanh \varepsilon \sinh u / \cosh \alpha .
$$

Finally, introducing $\cos \vartheta=\tanh \varepsilon / \tanh \alpha$ gives in the $h$-law of cosines

$$
\cosh \delta=\cosh u \cosh \alpha-\sinh \alpha \sinh u \cos \vartheta .
$$

In an exactly analogous way we find

$$
\cosh \alpha=\cosh \delta \cosh \beta-\sinh \delta \sinh \beta \cos \varphi .
$$

Now, introducing $\cos \vartheta=\tanh \varepsilon / \tanh \alpha$ into $\cos \varphi=\tanh (u-\varepsilon) / \tanh \delta$ results in

$$
\tanh \delta \cos \varphi=\frac{\tanh u-\tanh \alpha \cos \vartheta}{1-\tanh u \tanh \alpha \cos \vartheta}
$$

But, this should be a velocity composition law [cf. Fig. 5 where the triangle has to be fitted on a surface of a pseudosphere rather than the flat $e$-plane], and it will become one when we introduce the velocity components $\bar{u}_{1}=\bar{\alpha}=\tanh \alpha$, and $\bar{u}_{2}=\bar{\delta}=\tanh \delta$.

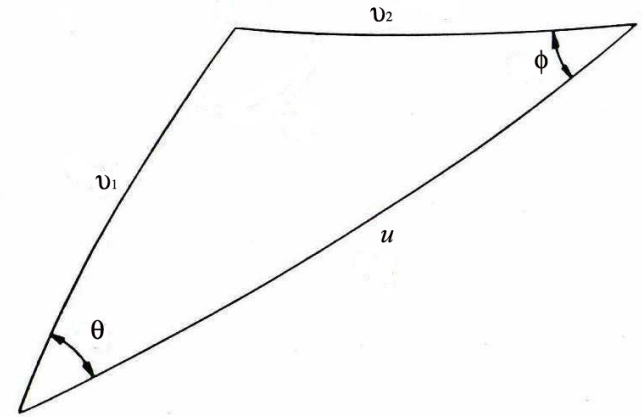

artwork/H-5.jpg

FIG. 5: Hyperbolic velocity triangle.

Introducing these definitions into (22) gives

$$
\bar{u}_{2} \cos \varphi=\frac{\bar{u}-\bar{u}_{1} \cos \vartheta}{1-\bar{u} \bar{u}_{1} \cos \vartheta} .
$$

In the limit as $\alpha, \gamma \rightarrow \infty, \bar{u}_{1}, \bar{u}_{2} \rightarrow 1$, and they become light signals.

The $h$-cosine law, $(20)$, can be written as

$$
\frac{\sinh \delta}{\sinh \alpha}=\frac{\tanh \delta}{\tanh \alpha} \cosh u(1-\tanh \alpha \tanh \beta \cos \vartheta)=\frac{\sin \vartheta}{\sin \varphi}
$$

which is the $h$-law of sines

$$
\frac{\sin \vartheta}{\sinh \delta}=\frac{\sin \varphi}{\sinh \alpha}
$$

showing that sides can be expressed in terms of angles in $h$-geometry [4]. The $h$-law of sines (25) happens also to be the equation of aberration [10]

$$
\bar{u}_{2} \sin \varphi=\frac{\bar{u}_{1} \sin \varphi \sqrt{ }\left(1-\bar{u}^{2}\right)}{1-\bar{u} \bar{u}_{1} \cos \vartheta}
$$


Taking the differential of $(23)$

$$
-\sin \varphi d \varphi=\frac{\bar{u}_{1}}{\bar{u}_{2}} \frac{\gamma^{-2} \sin \vartheta}{\left(1-\bar{u} \bar{u}_{1} \cos \vartheta\right)^{2}} d \vartheta
$$

and introducing (26) result in

$$
d \varphi=-\frac{\sqrt{ }\left(1-\bar{u}^{2}\right)}{1-\bar{u} \tanh \varepsilon} d \vartheta,
$$

where we used $\tanh \alpha \cdot \cos \vartheta=\tanh \varepsilon$. Dividing both sides by the time increment gives the Doppler shift as

$$
\nu=\mathfrak{D} \nu_{0},
$$

where

$$
\mathfrak{D}=\frac{\sqrt{ }\left(1-\bar{u}^{2}\right)}{1-\bar{u} \bar{u}_{1} \cos \vartheta}
$$

is the Doppler factor. A moving object that emits a signal at frequency $\nu_{0}=d \vartheta / d t$ with velocity $\bar{u}_{1}$, and $\nu=-d \varphi / d t$ is the frequency that the observer at rest registers.

If the signal is emitted at the velocity of light, $\bar{u}_{1}=1$, implying that $\alpha \rightarrow \infty$, and $\vartheta \rightarrow \pi / 2$, or equivalently $\varepsilon \rightarrow 0$, it follows from (20) that $\delta \rightarrow \infty$ such that the difference $\delta-\alpha$ remains finite

$$
e^{(\delta-\alpha)}=\cosh u=1 / \sin \vartheta
$$

where $\sin \vartheta$ is the ratio of smaller and larger concentric limiting arcs [6]. The Doppler shift (29) then becomes the exponential Doppler shift

$$
\nu=e^{-(\delta-\alpha)} \nu_{0}
$$

Ordinarily, one writes the Doppler factor (30) with $\bar{u}_{1}=1$ without realizing that it requires the limit $\alpha \rightarrow \infty$, which, in turn, requires that it be perpendicular to the motion. Then (23) and (26) can be combined in the half-angle formula, $\tan \varphi / 2=\sin \varphi /(1+\cos \varphi)$ to $\operatorname{read}[20]$

$$
\tan \varphi / 2=\left(\frac{1-\bar{u}}{1+\bar{u}}\right)^{1 / 2} \cot \vartheta / 2=e^{-u} \cot \vartheta / 2 .
$$

Equation (31) is the well-known expression for angle of parallelism: The ratio of concentric limiting arcs between two radii is the exponential distance between the arcs divided by the radius of curvature. Using the last equality in (33) we obtain the second equality in (31) when we set $\varphi=\pi / 2$. An observer in the frame in which the object is at rest will see it rotated by an amount $\sin \vartheta=\sqrt{ }\left(1-\bar{u}^{2}\right)$, exactly equal to the FL contraction [11]. Since the angle of parallelism provides a unique link between circular and $h$-functions, a rotation and contraction can only be related at the angle of parallelism, if the geometry is indeed hyperbolic. The equivalence of rotations and contractions was first discussed by Terrell [25] but his analysis cannot be extended to the situation where the angle is not acute, since the angle of parallelism must always be acute, tending to a right-angle only in the limit of $e$-geometry.

\section{KINEMATICS: $K$-CALCULUS}

With the realization that there is no such thing as a rigid body in relativity, Whitrow [28] went on to develop a radar method, or what he called a 'signal-function method' where light signals are transmitted between different inertial frames, and non-inertial ones. It was afterwards referred to as the ' $K$-calculus technique' by Bondi [1], although Milne [14] used it extensively in his research prior to him, and the original idea can be traced back to Robb [21].

\section{constant relative velocity: geometric-arithmetic mean equality}

As in kinematical relativity [14], time measurements are much more fundamental than distance measurements, the latter being deducible from the former. In other words, distances are measured by the elapse of time. This has 
been criticized by Born [3] as being impractical since no one has ever received light signals from nebulae beyond the horizon. However, it is far superior to the usual method in general relativity that uses a metric, or rigid ruler, to measure distance [1]. What was discarded in 'special' relativity made its come back in 'general' relativity.

The most ideal situation would be to introduce into the fabric of the theory distances measured in brightness, or the difference between apparent and absolute brightness. However, no one has ever succeeded in doing so and we will base all distance measurements on the so-called radar method [28], where a light signal is sent out and reflected at a later time. All that is needed is that at each reflection a certain retardation factor, $K$, comes in which is determined by the clock in the frame that is sending out the light pulse.

Consider two observers, $A$ and $B$, where observer $A$ sends out a light signal in his time $t_{1}^{A}$ which is received by observer $B$ in his time $t_{2}^{B}$. In terms of $A$ 's time, $B$ will receive it in time $K t_{1}^{A}$, where $K$ is some constant factor that is a function only of the relative velocity of the two inertial frames. The signal that passes $B$ in time $t_{2}^{B}$ will be reflected at some later time. The reflected signal passes $B$ in time $t_{3}^{B}$ which arrives at observer $A$ in time $t_{4}^{A}$, where $t_{4}^{A}=K t_{3}^{B}$. From this it is apparent that both observers will call the reflection time

$$
t^{r}=\sqrt{ }\left(t_{1}^{A} t_{4}^{A}\right)=\sqrt{ }\left(t_{2}^{B} t_{3}^{B}\right)
$$

which is the geometric mean of the time intervals, and it is an invariant independent of the frame. The appearance of the geometric mean, as opposed to the arithmetic mean, implies implicitly the existence of another time scale, namely, a logarithmic one [cf. eqn (66) below]. So the 'signal-function method' of Whitrow singles out the geometric mean as the time of reflection.

The reading shown by a synchronous (stationary) clock at the event should be midway between the observer's time, $t_{1}^{A}$, of sending out the signal, and the time he receives its reflection, $t_{4}^{A}$,

$$
t=\frac{1}{2}\left(t_{1}^{A}+t_{4}^{A}\right)
$$

This was Einstein's choice, but it by no means is the only choice [29]. The measure of the space interval is the difference between the "average" for the light-signalling process, (35), and the time the signal was sent out

$$
r=t-t_{1}^{A}=\frac{1}{2}\left(t_{4}^{A}-t_{1}^{A}\right) .
$$

In terms of $B$ 's coordinates, he will measure a time interval

$$
t^{\prime}=\frac{1}{2}\left(t_{2}^{B}+t_{3}^{B}\right)
$$

and a space interval

$$
r^{\prime}=\frac{1}{2}\left(t_{3}^{B}-t_{2}^{B}\right)
$$

separating the event from where he is located. The two systems of inertial coordinates $(t, r)$ and $\left(t^{\prime}, r^{\prime}\right)$ are related by

$$
\begin{array}{r}
t_{2}^{B}=t^{\prime}-r^{\prime}=K(t-r)=K t_{1}^{A} \\
t_{3}^{B}=t^{\prime}+r^{\prime}=K^{-1}(t+r)=K^{-1} t_{4}^{A} .
\end{array}
$$

The time $t_{2}^{B}$ is the time on $B$ 's clock when the signal is received, and $t_{3}^{B}$ is the moment on $B$ 's clock when it is sent back.

Suppose, for the moment, we place ourselves at the origin of $B$ 's frame. Then summing (39a) and (39b) gives

$$
t=\frac{1}{2}\left(t_{1}^{A}+t_{4}^{A}\right)=\frac{1}{2}\left(K+K^{-1}\right) t^{\prime}=\frac{t^{\prime}}{\sqrt{ }\left(1-\bar{u}^{2}\right)},
$$

showing that a clock traveling at a uniform velocity goes slower than one at rest. This expression for time-dilatation only holds for frames moving at a constant velocity $\bar{u}$ [cf. eqn (63) below].

In terms of the longitudinal Doppler shift, (4), the two system of coordinates are related by

$$
\begin{aligned}
t & =\frac{1}{2}\left(t_{1}^{A}+t_{4}^{A}\right)=\frac{1}{2}\left(K t_{3}^{B}+K^{-1} t_{2}^{B}\right) \\
& =\frac{1}{2}\left\{\left(K+K^{-1}\right) t^{\prime}+\left(K-K^{-1}\right) r^{\prime}\right\} \\
& =t^{\prime} \cosh u+r^{\prime} \sinh u,
\end{aligned}
$$


and

$$
\begin{aligned}
r & =\frac{1}{2}\left(t_{4}^{A}+t_{1}^{A}\right)=\frac{1}{2}\left(K t_{3}^{B}-K^{-1} t_{2}^{B}\right) \\
& =\frac{1}{2}\left\{\left(K-K^{-1}\right) t^{\prime}+\left(K+K^{-1}\right) r^{\prime}\right\} \\
& =t^{\prime} \sinh u+r^{\prime} \cosh u .
\end{aligned}
$$

These are none other than the well-known Lorentz transformations. Taking their differentials and forming the difference of their squares shows that the $h$-form

$$
d t^{2}-d r^{2}=d t^{\prime 2}-d r^{2}
$$

is invariant.

Now, let us ask what happens when the light-signal is reflected when it arrives at $B$. In this case, $t_{2}^{B}=t_{3}^{B} \equiv t^{r}$ is the time of reflection, and it occurs at the same point in space for $B$ so that $r^{\prime}=0$. The Lorentz transformations, (41) and (42), reduce to

$$
\begin{gathered}
t=t^{r} \cosh u \\
r=t^{r} \sinh u
\end{gathered}
$$

Equation (44a) is a statement of the arithmetic-geometric mean inequality: The arithmetic mean $t$ can never be inferior to the geometric mean $t^{r}$ since $\cosh u \geq 1$. Adding and subtracting the equations (44a) and (44b) give

$$
\begin{array}{r}
t+r=K t^{r} \\
t-r=K^{-1} t^{r} .
\end{array}
$$

Taking the differentials of (45a) and (45b), and then the product of the two, without requiring that $K$ be constant, result in

$$
d t^{2}-d r^{2}=d t^{r 2}-t^{r 2} d u^{2}
$$

A space-time interval has been transformed into a velocity space-time interval.

\section{constant relative acceleration}

It is generally acknowledged that acceleration has no effect on the rate of a clock [15], and that the expression for time-dilatation (40) can be used in its infinitesimal form whether or not $\bar{u}$ is constant. However, according to Einstein's equivalence principle uniform acceleration is equivalent to, or indistinguishable from, a uniform gravitational field. It has been shown from the gravitational red-shift that the latter, indeed, has an effect on the rate of a clock. This contradiction has been clearly pointed out by Whitrowl [29], who shows that the time-dilatation is greater when the velocity is varying with time than when it is constant. We convert his inequality into an equality.

For a particle under the influence of a constant gravitational acceleration

$$
g=\frac{d}{d t}\left(\frac{\bar{u}}{\sqrt{ }\left(1-\bar{u}^{2}\right)}\right)
$$

will be constant so that integration gives simply

$$
g t=\frac{\bar{u}}{\sqrt{ }\left(1-\bar{u}^{2}\right)}=\sinh u .
$$

Now, the velocity can be written as

$$
\bar{u}=\tanh u=\frac{g t}{\sqrt{ }\left(1+(g t)^{2}\right)}=\frac{d r}{d t} .
$$

If we further assume that $r=0$ at $t=0$, we get a second integral

$$
g r=\sqrt{ }\left(1+(g t)^{2}\right)-1=\cosh u-1 .
$$


This is the one-dimensional $h$-motion found by Born [2] in 1909, and by Sommerfeld [24] a year later. It will be our prototype of a one-dimensional system at constant acceleration.

Dividing (48) by (50) leads to the average velocity

$$
r / t=\tanh (u / 2)
$$

Consider two observers receding from one another with an average velocity $r / t$. Their identical clocks were synchronized at $t^{A}=t^{B}=0$ when they were at the same point. At time $t_{1}^{A}$, A emits a signal which is picked up and immediately reflected by $\mathrm{B}$ at time $t^{B r}$, and received back at $\mathrm{A}$ at time $t_{3}^{A}$. The space interval is

$$
t-t_{1}^{A}=t_{3}^{A}-t=\frac{1}{2}\left(t_{3}^{A}-t_{1}^{A}\right)=r .
$$

From this it follows that

$$
\begin{array}{r}
t_{1}^{A}=t-r \\
t_{3}^{A}=t+r \\
t^{A r}=\sqrt{ }\left(1-(r / t)^{2}\right) t .
\end{array}
$$

Since the Doppler shift is

$$
K=\left(\frac{1+\bar{u}}{1-\bar{u}}\right)^{1 / 2}=\left(\frac{1+r / t}{1-r / t}\right)
$$

we can express $(52 \mathrm{a})$ and $(52 \mathrm{~b})$ as $t_{3}^{A}=K t_{1}^{A}$, or

$$
\begin{aligned}
& t^{B r}=K^{1 / 2} t_{1}^{A}, \\
& t_{3}^{A}=K^{1 / 2} t^{B r} .
\end{aligned}
$$

But, from (52c) it is apparent that $t^{B r}=t^{A r}$ so that the clocks remain synchronized, and we can drop the superscripts on the time.

Expressing $r$ and $t$ in terms of $t_{1}$ and $t_{3}$ in (50) we find [17]

$$
g=2 \frac{r}{t^{r 2}}=\frac{1}{t_{1}}-\frac{1}{t_{3}}
$$

Employing (54a) and (54b) we write (55) as

$$
g=\frac{K^{1 / 2}-K^{-1 / 2}}{t^{r}}=\frac{2 \sinh (u / 2) \cosh (u / 2)}{t}
$$

which is identical to (48).

Equation (55) enables us to express the Doppler shift, $K$, in terms of the ratio of the time the signal was received back to that when it was sent out

$$
t_{3} / t_{1}=K
$$

Taking the logarithms of both sides of (57), and then differentiating with respect to $t$, give

$$
\frac{d \ln t_{3}}{d t}-\frac{d \ln t_{1}}{d t}=\frac{1+\bar{u}}{t_{3}}-\frac{1-\bar{u}}{t_{1}}=\frac{1}{1-\bar{u}^{2}} \frac{d \bar{u}}{d t}
$$

where we have used the differentials of $(52 \mathrm{a})$ and $(52 \mathrm{~b})$. Dividing both sides by $\sqrt{ }\left(1-\bar{u}^{2}\right)$ results in

$$
\frac{K}{t_{3}}-\frac{K^{-1}}{t_{1}}=\frac{1}{\left(1-\bar{u}^{2}\right)^{3 / 2}} \frac{d \bar{u}}{d t}=g,
$$

which is identical to (55). If the ratio (57) had been proportional to the square of the Doppler shift, we would have found that (58) vanishes. 
If we consider $t^{\prime}$ to be the time of reflection on $B$ 's clock, we can write (55) as

$$
\begin{gathered}
\frac{1}{t_{1}}=\frac{1}{t^{\prime}}+g / 2 \\
\frac{1}{t_{3}}=\frac{1}{t^{\prime}}-g / 2 .
\end{gathered}
$$

Adding (59a) and (59b) shows that the time of reflection on B's clock is the harmonic mean

$$
\frac{1}{t^{\prime}}=\frac{1}{2}\left(\frac{1}{t_{1}}+\frac{1}{t_{3}}\right)
$$

in contrast with the geometric mean as the time of reflection for uniform motion. The difference between (59a) and (59b) is (55). Writing $t_{1}=t-r$ and $t_{3}=t+r$ in (60) clearly shows that the space-time interval is not invariant

$$
t^{2}-r^{2}=t^{\prime} t=t^{r 2}
$$

unless we require the reflection times to be the same, meaning that clocks $A$ and $B$ are synchronous [19].

Multiplying the left- and right-hand sides of (59a) and (59b), rearranging, and then taking the square roots give

$$
t^{\prime}=\operatorname{sech}(u / 2) t^{r}=\operatorname{sech}^{2}(u / 2) t,
$$

where the second equality follows from (52c). Equations (61) give quantitative relationships to the statements that the harmonic mean is always smaller than the geometric mean which is smaller than the arithmetic mean, because the equality of times can never apply. The first equality in equation (61) states physically that the time of reflection on $B$ 's clock is always less than on $A$ 's clock.

From (59a) and (59b) it also follows that

$$
K=\frac{t_{3}}{t_{1}}=\frac{1+g t^{\prime} / 2}{1-g t^{\prime} / 2}
$$

A comparison of (53) and (62) leads to

$$
\frac{1}{2} g t^{\prime}=r / t
$$

Differentiating with respect to the arithmetic time average gives

$$
d t=\cosh ^{2}(u / 2) \frac{d t^{\prime}}{\sqrt{ }\left(1-\bar{u}^{2}\right)} .
$$

In comparison with the expression for time-dilatation for uniform motion, (40), expression (63) is larger for uniform acceleration. Uniform acceleration of $B$ does, indeed, affect the apparent rate according to $A$ of a clock carried by $B$ [29].

The transformation laws (54a) and (54b) can be expressed as

$$
\begin{gathered}
t+r=K^{1 / 2} t^{r} \\
t-r=K^{-1 / 2} t^{r} .
\end{gathered}
$$

Taking the differentials of (64a) and (64b), and then their product, result in

$$
\begin{aligned}
d s^{2}: & =d t^{2}-d r^{2}=d t^{r 2}-\frac{1}{4} t^{r 2}(d K)^{2} / K^{2} \\
& =d t^{r 2}-\frac{1}{4} t^{r 2} d u^{2} .
\end{aligned}
$$

The appearance of $t^{r}$ in the velocity space component of the metric (65) implies uniform expansion. Introducing the logarithmic time

$$
\tau=2 \tau_{0} \ln \left(t^{r} / \tau_{0}\right),
$$

where $\tau_{0}$ is an absolute constant, enables (65) to be written as

$$
d s^{2}=d t^{2}-d r^{2}=\frac{e^{\tau / \tau_{0}}}{4}\left\{d \tau^{2}-\tau_{0}^{2} d u^{2}\right\}
$$


Thus, the formulas of the transformation of coordinates (64a) and (64b) can be written as

$$
\begin{aligned}
& t+r=\tau_{0} e^{\tau / \tau_{0}+u / 2} \\
& t-r=\tau_{0} e^{\tau / \tau_{0}-u / 2}
\end{aligned}
$$

At constant $t^{r}$, a surface of revolution is obtained by rotating the hyperbola $t^{2}-r^{2}=t^{r}$ around the $t$ axis to give a bowl shaped form.

The equivalence relations $(68 \mathrm{a})$ and $(68 \mathrm{~b})$ can be combined to read

$$
t+r=K(t-r)
$$

Comparing this with the case of constant velocity

$$
\begin{array}{r}
t+r=K\left(t^{\prime}-r^{\prime}\right) \\
t-r=K^{-1}\left(t^{\prime}+r^{\prime}\right),
\end{array}
$$

we conclude that the former does not retain its invariant hyperbolic form whereas the latter does

$$
t^{2}-r^{2}=t^{\prime 2}-r^{2}
$$

Adding and subtracting the equations yields the well-known Lorentz transformations (41) and (42), and from which it can be concluded that the Lorentz transformations leave invariant the hyperbolic 'distance', (71).

In terms of radar measurements, (69) consists in a single observer: A light pulse is emitted in time $t_{1}$, and observed by him at a later time $t_{2}=K t_{1}$. Alternatively, in the case of constant velocity, (70a) says a light signal is emitted at time $t_{1}^{\prime}$, in the prime inertial frame, and observed in the unprimed frame at a later time $t_{2}=K t_{1}^{\prime}$. Whereas, $(70 \mathrm{~b})$ says that if a signal is emitted at time $t_{1}$, it will be observed at time $t_{2}^{\prime}$ in the primed inertial frame.

For uniform motion the geometric mean time remains invariant, $\sqrt{ }\left(t_{1} t_{2}\right)=\sqrt{ }\left(t_{1}^{\prime} t_{2}^{\prime}\right)$. This is the same as requiring the $h$-line element (71) to be invariant. While, for uniform acceleration, the time of reflection in the $B$ frame is the harmonic mean of the $A$ frame. In his analysis of uniform acceleration, Page [17] attempted to show that the space-time interval between neighboring points is not constant. His analysis replaces (59b) by

$$
\frac{1}{t_{3}}=\frac{1}{t^{\prime \prime}}-g / 2
$$

This condition would necessarily imply that the harmonic means in the two frames are equal. Solving (59a) and (72) for the times $t^{\prime}$ and $t^{\prime \prime}$, with $t^{\prime \prime}>t^{\prime}$ we get

$$
\begin{array}{r}
\tilde{t}:=\frac{1}{2}\left(t^{\prime}+t^{\prime \prime}\right)=t \operatorname{sech}^{2}(u / 2) \\
\tilde{r}:=\frac{1}{2}\left(t^{\prime \prime}-t^{\prime}\right)=[r-t \tanh (u / 2)] \operatorname{sech}^{2}(u / 2) .
\end{array}
$$

However, consulting (51), (73b) vanishes, and hence $\tilde{r}=0$. The time of reflection is given by the harmonic mean (60). Therefore, for uniformly accelerating systems the point of reflection must occur at the origin of $B$ 's frame, whose time is given by the harmonic mean of $A$ 's clock.

\section{MULTI-DIMENSIONAL LOBACHEVSKY VELOCITY SPACE}

The one-dimensional velocity space of the previous subsection can be generalized to at least two velocities by realizing that (49) is a Beltrami coordinate for the velocity. A second Beltrami coordinate can be defined similarly

$$
\bar{v}=\tanh v
$$

The Weierstrass coordinates can now be introduced as

$$
X=\bar{u} T, \quad Y=\bar{v} T, \quad \text { and } \quad T=\cosh u \cosh w,
$$

where $u, v w$, and $z$ are four sides of a Lambert quadrilateral, shown in Fig. 6, consisting of three right-angles and one acute angle between $w$ and $z$. 


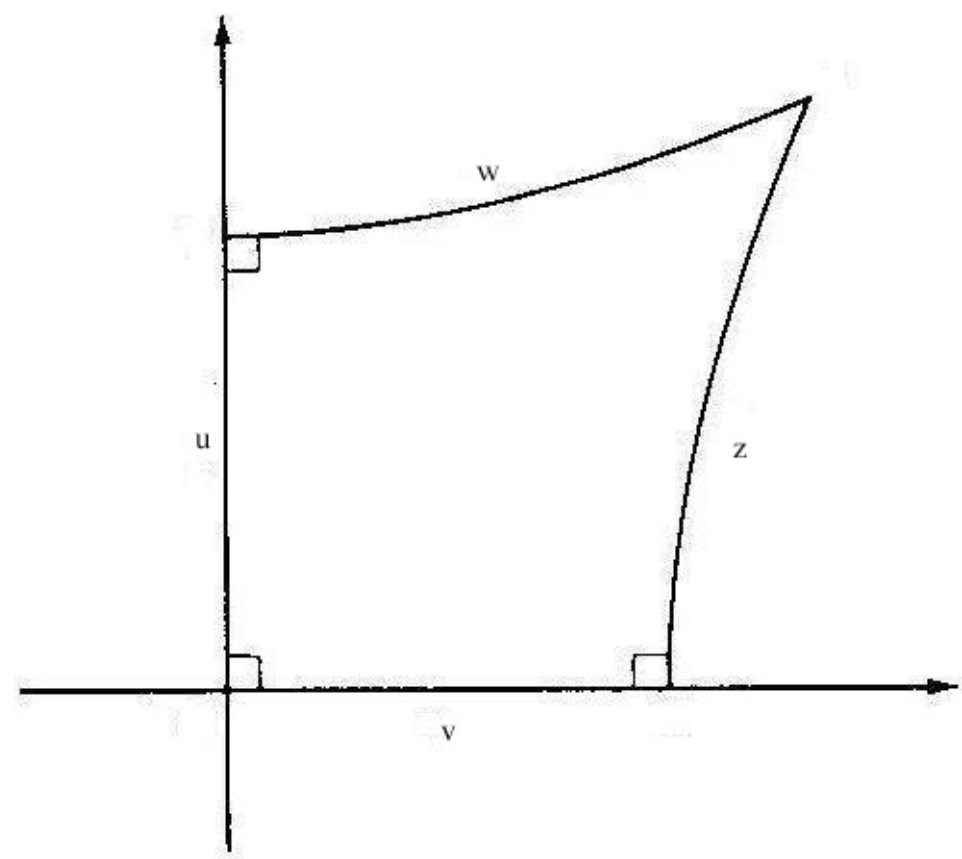

artwork/H-6.jpg

FIG. 6: A Lambert quadrilateral in velocity space considering of three right-angles and one acute angle.

The condition that the two sides will intersect to form an acute angle is

$$
1-\tanh ^{2} u-\tanh ^{2} v=1-\bar{u}^{2}-\bar{v}^{2}>0 .
$$

The $e$-measures of the two sides are $\bar{w}=\bar{v} / \sqrt{ }\left(1-\bar{u}^{2}\right)$, and $\bar{z}=\bar{u} / \sqrt{ }\left(1-\bar{v}^{2}\right)$. By giving to each point the triple $(X, Y, T)$ of Weierstrass coordinates, the hyperbolic plane is mapped on to the locus

$$
T^{2}-X^{2}-Y^{2}=1
$$

which is one of two sheets of a hyperboloid in Cartesian three-dimensions. The infinitesimal metric

$$
d X^{2}+d Y^{2}-d T^{2}=d w^{2}+\cosh ^{2} w d u^{2}=\frac{\left(1-\bar{v}^{2}\right) d \bar{u}^{2}+2 \bar{u} \bar{v} d \bar{u} d \bar{v}+\left(1-\bar{u}^{2}\right) d \bar{v}^{2}}{\left(1-\bar{r}^{2}\right)^{2}}
$$

where $\bar{r}^{2}=\bar{u}^{2}+\bar{v}^{2}$, is the spatial component of the Lobachevsky velocity space metric. In order to derive the full time-velocity space metric, we magnify the $T$ coordinate, $t^{r}>0$ times, viz., $T=t^{r} / \sqrt{ }\left(1-\bar{r}^{2}\right)$ so as to obtain the time-like, indefinite metric as

$$
d s^{2}=d T^{2}-d X^{2}-d Y^{2}=d t^{r 2}-t^{r 2}\left(d w^{2}+\cosh ^{2} w d u^{2}\right) .
$$

A time-velocity metric, similar to (67), was derived by Friedmann in 1922 using the Einstein equations to relate the coordinates to the Lagrangian variables, $u_{i}$. It was derived under the condition that matter was "dust-like" exerting zero pressure. It was also assumed that the velocities variables $u_{i}$ are constants relating the spatial coordinates $x_{i}$ to time, but, subsequently, they were differentiated to obtain the Lobachevsky-Friedmann metric (67) [7].

As can be seen from the definition of the Weierstrass coordinates, (75), each of the coordinates become magnified $t^{r}$ times [7, eqn (94.47)]. In a multi-dimensional velocity space, the spatial part of the metric can be written as

$$
d \sigma^{2}=\frac{(d \vec{u})^{2}-(\vec{u} \times d \vec{u})^{2}}{\left(1-\vec{r}^{2}\right)^{2}}
$$


by introducing the coordinates $X_{i}=\bar{u}_{i} t^{r} / \sqrt{ }\left(1-\bar{r}^{2}\right)$ into the infinitesimal metric

$$
d s^{2}=d T^{2}-\sum_{i} X_{i}^{2}
$$

In relation to the Robertson metric, the scale factor $R(t)$ multiplying the spatial part of the metric is just $t$, which implies uniform expansion. Introducing logarithmic time according to

$$
\tau=\tau_{0} \ln \left(t^{r} / \tau_{0}\right)
$$

(and not (66)) into (78) gives

$$
d s^{2}=e^{2 \tau / \tau_{0}}\left\{d \tau^{2}-\tau_{0}^{2}\left(d w^{2}+\cosh ^{2} w d u^{2}\right)\right\}
$$

The proper time interval is the quantity $s_{0}$ determined at constant velocity by the equation

$$
s_{0}=\int_{0}^{\tau} e^{\tau / \tau_{0}} d \tau=\tau_{0}\left(e^{\tau / \tau_{0}}-1\right)
$$

This law could have been anticipated because $t^{r}$ is the geometric mean. Only for $\tau \ll \tau_{0}$ will the proper time coincide with $\tau$. The exponential variable scale factor multiplies both time and velocity increments, and testifies to the fact that they are not independent, but, are related by the Beltrami coordinates and logarithmic time.

For fixed $s_{0}$ the velocity space line element is

$$
d \sigma^{2}=\tau_{0}^{2} e^{2 \tau / \tau_{0}}\left(d w^{2}+\cosh ^{2} w d u^{2}\right)
$$

The terms in the parentheses have the metric form of a pseudosphere in velocity space, with constant negative curvature, -1 . The scale factor is the same exponential that appears in the proper time increment. The hallmark of a pseudosphere is that lines which do not intersect are, nevertheless, not parallel. Along a light track (80) vanishes resulting in

$$
d \tau=\tau_{0} \frac{\sqrt{ }\left\{\left(1-\bar{u}^{2}\right)^{2} d \bar{w}^{2}+\left(1-\bar{w}^{2}\right) d \bar{u}^{2}\right\}}{\left(1-\bar{u}^{2}\right)\left(1-\bar{w}^{2}\right)} .
$$

This is a generalization of the well-known one-dimensional expression, whose integral identifies (1) as the length of the corresponding segment of a Lobachevsky straight line.

\section{LIMITING CASE OF A LAMBERT QUADRILATERAL: UNIFORM ACCELERATION}

A limiting case arises when inequality (76) reduces to an equality

$$
\bar{u}^{2}+\bar{v}^{2}=1
$$

or $\bar{v}=\sqrt{ }\left(1-\bar{u}^{2}\right)=: \bar{u}^{*}$. The velocities $\bar{u}$ and $\bar{u}^{*}$ are said to be complementary [8]. The defining relation for uniform acceleration is (53), which upon resolving for the velocity gives

$$
\bar{u}=\tanh u=\frac{2(r / t)}{1+(r / t)^{2}}
$$

$r / t$ represents the Euclidean 'length,' while $u$ the Poincaré length in the Poincaré model; the two being related by

$$
e^{u}=\frac{1+(r / t)}{1-(r / t)} .
$$

The complementary velocity is found to be

$$
\bar{u}^{*}=\frac{1-(r / t)^{2}}{1+(r / t)^{2}}=\operatorname{sech} u=\sqrt{ }\left(1-\bar{u}^{2}\right),
$$

which verifies (82). 
The angle of parallelism, (10), in the present context, is

$$
\Pi\left(u^{*}\right)=2 \tan ^{-1} e^{-u^{*}}
$$

which is defined solely in terms of the 'distance' $u^{*}$ from the foot of the perpendicular to the angle of parallelism. The angle of parallelism is the lower bound for the angle of parallax. It was Bernoulli who first showed that

$$
2 \tan ^{-1} e^{-u^{*}}=\frac{1}{i} \ln \left(\frac{1+i e^{-u^{*}}}{1-i e^{-u^{*}}}\right) .
$$

In particular,

$$
r / t=\tanh (u / 2)=\tan \left[\Pi\left(u^{*}\right) / 2\right]=e^{-u^{*}}
$$

shows that the closer the complementary velocity $u^{*}$ is to zero, the closer $\Pi$ is to being a right-angle. For large $u^{*}$, or non-relativistic velocities, the angle of parallelism is practically zero. For the earth's orbital motion $(87)$ is $10^{-4}$, giving an angle of parallelism $\Pi=89^{0} 59^{\prime} 39^{\prime \prime} .4$. The deviation from $e$-space is only $20^{\prime \prime} .6$. However, things change drastically as the velocity of light is approached: For a relative velocity 0.95 , the angle of parallelism drops to $18^{0} 12^{\prime}$, and vanishes in the limit [22].

The double angle formula

$$
\tan \Pi\left(u^{*}\right)=1 / \sinh u^{*}=\sinh u
$$

shows that $\Pi$ provides the link between circular and $h$-functions. In particular, (88) relates the angle of parallelism to the particle velocity [cf. eqn (48)].

Consider a Lambert quadrilateral with three right-angles and an ideal point in Fig. 7.

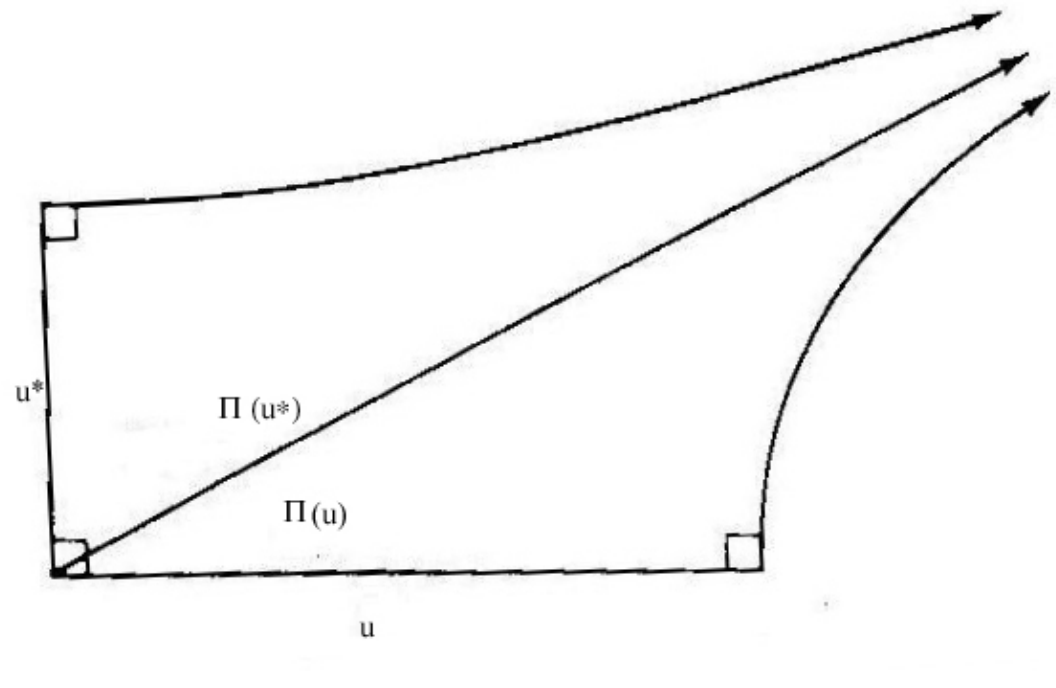

FIG. 7: A Lambert quadrilateral comprised of complementary segments where the 'fourth vertex' is an ideal point.

The opposite right angle is divided into two angles of parallelism such that

$$
\Pi(u)+\Pi\left(u^{*}\right)=\pi / 2 .
$$

Using Bernoulli's relation, (86), (89) implies that the complementary velocities, $u$ and $u^{*}$, which are adjacent to the two angles of parallelism, are related by

$$
e^{-u^{*}}=\left(\frac{1-e^{-u}}{1+e^{-u}}\right)
$$


Equation (84) implies the addition law for the $h$-measure of the complementary velocities, which, in turn, implies the product law for the average velocities.

Rather, if $u_{1}$ and $u_{2}$ are the components of the $h$-measure of the velocity $u$, their composition law follows Poincaré's addition law

$$
e^{-u}=\frac{e^{-u_{1}}+e^{-u_{2}}}{1+e^{-u_{1}-u_{2}}}
$$

Introducing (87) into (84), and then into (90) lead to the result that

$$
\tanh (u / 2)=\prod_{i=1}^{n} \tan \left[\Pi\left(u_{i}^{*}\right) / 2\right]=e^{-\sum_{i=1}^{n} u_{i}^{*}}=\hat{\ell}^{n},
$$

where the $e$-length $\hat{\ell}$ is the geometric mean of a sample of size $n$.

We conclude that whereas uniform motion utilizes the arithmetic mean, uniform acceleration singles out the geometric mean. For uniform motion, the geometric mean time is the invariant reflection time, while the harmonic mean time is the reflection time for uniform acceleration.

\section{ADDITIVITY OF THE RECESSION AND DISTANCE IN HUBBLE'S LAW}

The fact that the shift $z=\delta \lambda / \lambda_{0}$ for lines in the spectrum of a given galaxy is independent of the wavelength is a necessary, but not a sufficient condition, that the red-shift is due to motion. It was Hubble who interpreted these red-shifts as Doppler shifts, indicative of recessional motion. In so doing he obtained a linear relation between the velocity of recession, $u$, and radial distance, $r$, with a constant of proportionality that is the same for all galaxies. We will show that both these quantities are additive.

There will be a red-shift if the detected wavelength, $\lambda$, is greater than the emitted wavelength, $\lambda_{0}$, in

$$
1+z=\frac{\lambda}{\lambda_{0}}=K=e^{u}
$$

Now, $K$ is the ratio of the received, $t_{2}$, to the emitted time, $t_{1}$. We can therefore define a $h$-measure of the time interval as [14]

$$
\tau / \tau_{0}=\ln \frac{t_{2}}{t_{1}}=\ln K
$$

A comparison of (92) and (93) results in

$$
u=\frac{\tau}{\tau_{0}}=H r
$$

where $u$ is the $h$-measure of the velocity, $H=\tau_{0}^{-1}$, the Hubble parameter, and $r=\tau$ is the $h$-measure of distance. Hubble's law (94) could have also been derived by setting the one-dimensional velocity space metric (65) equal to zero, and introducing the logarithmic time (79).

Consequently, (92) is the exponential law [19]

$$
1+z=e^{H \tau}
$$

Only when $H \tau \ll 1$ can we neglect powers of $H \tau$ greater than first so that (95) reduces to the relation [9]

$$
z=H \tau \text {. }
$$

The exponential law (95) implies that when there is more than one red-shift, it is their geometric mean which should be taken. For example, the cluster Group II has $n=21$ redshifts, in which case (92) generalizes to

$$
\prod_{i=1}^{n}\left(1+z_{i}\right)=\prod_{i=1}^{n} \frac{\lambda_{i}}{\lambda_{0 i}}=\prod_{i=1}^{n} K\left(u_{i}\right)=\exp \left(\sum_{i=1}^{n} u_{i}\right) .
$$

$H$-velocities, $u_{i}$, like the $h$-distances $r_{i}$, are additive. The average wavelength is the geometric mean wavelength. This is implied by the exponential law (97). 


\section{EXPONENTIAL RED-SHIFT}

Let us again consider signal transmission from observers $A$ and $B$. We know that observer $B$ will receive a signal sent from $A$ in time $t_{2}^{A}=K t_{1}^{A}$. If we further specify that $A$ will receive back the signal in a time interval increased $K$ times again, it means that $B$ reflects the signal upon receiving it because $t_{2}^{B}=t_{3}^{B}$.

What we want to show now is that the frequency shift, (29), is the inverse of the ratio of the arithmetic times, and give a geometrical interpretation of the result. We claim that

$$
\frac{\nu}{\nu_{0}}=\frac{t_{2}^{B}+t_{3}^{B}}{t_{1}^{A}+t_{4}^{A}}=K \frac{1+K^{-2}\left(t_{4}^{A} / t_{1}^{A}\right)}{1+t_{4}^{A} / t_{1}^{A}}=\mathfrak{D},
$$

where we are allowing for signals other than light since $\bar{u}_{2}$ need not be unity. The condition that (98) hold is

$$
\frac{t_{4}^{A}}{t_{1}^{A}}=\left(\frac{1+\bar{u}_{2} \cos \varphi}{1-\bar{u}_{2} \cos \varphi}\right)\left(\frac{1+\bar{u}}{1-\bar{u}}\right)=\frac{1+\bar{u}_{1} \cos \vartheta}{1-\bar{u}_{1} \cos \vartheta},
$$

where the second equality results from the velocity addition formula. If light is being used as the messenger, $\bar{u}_{1}=$ $\bar{u}_{2}=1$. From the definition of the cross-ratio (3), which expresses distances as ratios instead of differences, the $h$-length of an arc subtended by angles $\varphi$ and $\vartheta$, with $\varphi>\vartheta$ is [13]

$$
r / r_{0}=\frac{1}{2} \ln \frac{(1+\cos \vartheta)(1-\cos \varphi)}{(1-\cos \vartheta)(1+\cos \varphi)}
$$

The second formula of Lobachevsky relates the exponential distance between two horocycles, $\varrho$, to the $h$-cosine of the $h$-distance, $r$, [cf. eqn (31)] viz.,

$$
e^{\varrho / \kappa}=\cosh \left(r / r_{0}\right)=1 / \sin \vartheta,
$$

where $\kappa$ is an absolute constant. Introducing (101) into the red-shift formula (98) gives

$$
\nu=e^{-\varrho / r_{0}} \nu_{0} .
$$

An exponential law for the longitudinal red-shift, (102), has already been proposed [19, §6.4], but not for the transverse red-shift. The linear approximation

$$
\frac{\Delta \nu}{\nu_{0}} \simeq \frac{\varrho}{\kappa}
$$

is usually quoted in texts on cosmology because the radius of curvature $\kappa$ is very large compared to $\varrho$. In general, the ratio $\varrho / \kappa$ will be of the order of the ratio of the Schwarzschild radius to the radius of a star, again a very small quantity. Therefore, non-Euclidean space time should manifest itself on small scales of the order of the Schwarzschild radius since the larger $\kappa$ is, in comparison to $\varrho$, the less non-Euclidean character of the Lobachesvkian plane.

\section{COMPARISON TO 'GENERAL' RELATIVITY}

Einstein's theory of relativity essentially consists of two principles [7, p. 233]: The unification of space and time into a four dimensional space with an indefinite metric, and the relation of the curvature of the space to the presence of matter. Einstein also proposed an 'equivalence' principle between inertia and gravitational mass, or between acceleration and gravitation. The latter has been criticized by Fock [7, pp. 232-233], and by the present writer [10]. Gravitational considerations appear only in the specification of the absolute constant, which is related to the constant, negative curvature of the hyperbolic space. Said differently, the centrifugal term appears explicitly in the metric, while the gravitational potential does not.

In the general case of non-uniform motion the relevant space is the Friedmann-Lobachevsky velocity space [7, §94], which we have derived here without any appeal to Einstein's equations, and the unphysical assumption that matter must be 'dust like' at zero pressure. The velocity components are related to the sides of a Lambert quadrilateral whose Weierstrass coordinates of the point of the acute angle show that the geometric mean time enters as a magnification of these coordinates, and not as a separate entity. Most importantly, by avoiding the 'rigid scaffolding' employed by Einstein, which is applicable to inertial frames of reference only [7], acceleration has been accounted for as changes in velocity space, where the independence of the 'coordinates' and time has disappeared. 


\section{CONCLUSIONS}

We have made a synthesis of Poincaré's model of the $h$-plane and his, and others, contributions to relativity. The key has been shown to be the relation between the longitudinal Doppler shift and the cross-ratio which measures $h$-distances. According to de Broglie [5], Poincaré

had a somewhat skeptical attitude towards physical theories, and thought there was generally an infinity of different viewpoints and different ideas, all logically equivalent, from which the scientist only chooses for reasons of convenience.

Yet, it is hard to reconcile that:

- Poincaré discovery in 1883 that the most general Möbius transformation of the complex plane $\mathcal{M}(z)=(a z+$ $b) /(c z+d)$ could not be carried over to the general Lorentz transformation, expressed in homogeneous coordinates as a Möbius transformation, which he coined the name some twenty-two years later [18];

- the uniform state of motion of relative velocity $\lambda$ is the composed map from the Poincaré disc model to the hemisphere back to the Klein model, and, in doing so, straightens out every circle orthogonal to the unit circle into a straight line segment, thereby straightening out bowed geodesics into straight lines at the cost of losing conformality;

- the angle of parallelism, which provides a link between circular and $h$-functions, is related to viewing the FL contraction as a rotation orthogonal to the direction of the motion.

With the basic realization that the underlying geometry is hyperbolic, there is no longer any need of the artificial distinction between the 'special' and 'general' theories of relativity.

[1] Bondi H (1960) Cosmology. Cambridge U P, London

[2] Born M (1909) Ann. d. Physik 30: 1

[3] Born M (1943) Experiment and Theory in Physics. Cambridge U P, London

[4] Busemann H, Kelly, P J (1953) Projective Geometry and Projective Metrics. Academic Press, New York

[5] de Broglie L (1951) Savants et découvertes. Albin Michel, Paris

[6] Faber R L (1983) Foundations of Euclidean and Non-Euclidean Geometry. Marcel Dekker, New York

[7] Fock V (1969) The Theory of Space, Time and Gravitation, 2nd ed. Pergamon Press, Oxford

[8] Greenberg M J (1993) Euclidean and Non-Euclidean Geometries: Development and History, 3rd ed. W.H. Freeman, New York

[9] Hoyle F, Burbidge G, Narlikar J V (2000) A Different Approach to Cosmology. Cambridge U P, Cambridge

[10] Lavenda B H (2008) "On the equivalence of fields of acceleration and gravitation, submitted to Naturwissenschaften

[11] Lavenda B H (2008) "Aberration and radiation pressure in the Klein and Poincaré models" arXiv:0806.2772

[12] Lobachevsky N I (1898) Zewi geometrische Abhandlungen. Leipzig

[13] Meschkowski H (1964) Noneuclidean Geometry. Academic Press, New York

[14] Milne E A (1948) Kinematical Relativity. Oxford U P, London

[15] Møller C (1952) Theory of Relativity. Oxford U. P., London

[16] Needham T (1997) Visual Complex Analysis. Clarendon Press, Oxford

[17] Page L (1936) Phys Rev 49:254-268.

[18] Poincaré H (1905) Comptes rendus hebdomadaires des seances de l'Academie des Sciences. 140: 1504-1508.

[19] Prohovnik S J (1967) The Logic of Special Relativity. Cambridge U P, London

[20] Rindler W (1982) Introduction to Special Relativity. Clarendon Press, Oxford

[21] Robb A A (1911) Optical Geometry of Motion. W. Heffer \& Sons, Cambridge

[22] Silberstein L (1914) The Theory of Relativity. MacMillan, London

[23] Sommerfeld A (1909) Verh. deutsch. Phys. Ges. XI:577

[24] Sommerfeld A (1910) Ann. d. Physik 33: 670

[25] Terrell J (1959) Phys Rev 116: 1041

[26] Ungar A A (2008) Analytic Hyperbolic Geometry and Albert Einstein's Special Theory of Relativity. World Scientific, Singapore

[27] Varićak V (1910) Phys. Zeitschrift. XI: 93, 287, 586; (1912) Jahresberich der deutschen Math. Vereinigung. XXI: 103

[28] Whitrow G J (1933) Q J Math (Oxford) 4: 161

[29] Whitrow G J (1980) The Natural Philosophy of Time, 2nd ed. Clarendon Press, Oxford 